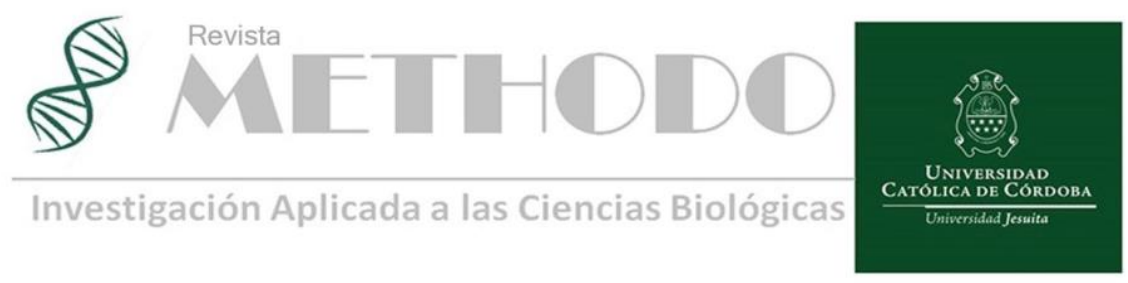

\title{
Identificación y expresión del efector central fúngico Pep1 en Thecaphora frezii
}

\section{Identification and expression of the fungal central effector Pep1 in Thecaphora frezii}

\author{
Néstor Walter Soria ${ }^{1}$ (D) , Pablo Yang ${ }^{1}$ (D) , María Soledad Díaz ${ }^{2}$ (iD), Ana Cristina Figueroa ${ }^{2}$ (iD), Valeria \\ Roxana Alasino ${ }^{2,3}$ (D), Dante Miguel Beltramo ${ }^{1,2,3}$. \\ 1 Universidad Católica de Córdoba, Facultad de Ciencias Químicas, Cátedra de Biotecnología, Unidad Asociada al CONICET: Área de Cs. Agrarias, Ingeniería, \\ Cs. Biológicas. \\ 2 Centro de Excelencia en Productos y Procesos de Córdoba - CEPROCOR \\ 3 CONICET \\ Correspondencia: Néstor Walter Soria, e-mail: nestorwsoria@gmail.com
}

\section{Resumen}

Thecaphora frezii es un hongo fitopatógeno perteneciente a la clase Ustilaginomicetes, que produce la enfermedad del carbón de maní. En su ciclo biológico presenta tres estructuras, las teliosporas (es la estructura de resistencia) y las basidiosporas e hifas. El micelio (hifas) es la estructura infectiva, que penetra en el ginóforo de la planta e inicia la infección. Para dicha acción, sería necesaria la expresión de la proteína Pepl ya que la misma fue identificada en otros Ustilaginomicetes como el Ustilago maydis y el Ustilago hordei, que infectan al maíz y a la cebada, respectivamente, y su expresión es fundamental para dicha acción.

Pudimos amplificar el ADN copia de Pepl de Thecaphora frezii cuya secuencia traducida codificaría para una proteína de 180 aminoácidos. Se observaron grandes homologías con ortólogas de otras especies y la presencia de cuatro cisteínas conservadas. Paralelamente, medimos los niveles de expresión de este transcripto, encontrándose muy elevado en las hifas, coincidiendo con el estadio infectivo del hongo.

Futuros estudios funcionales de inactivación génica del gen pepl deberán realizarse para comprobar fenotípicamente el efecto que este gen provoca en cultivos de maní.

Palabras claves: Thecaphora frezii; Pepl; maní; infección

\begin{abstract}
Thecaphora frezii is a phytopathogenic fungus belonging to the Ustilaginomycetes class, which causes the peanut smut disease. In its biological cycle it has three structures, teliospores (is the resistance structure), basidiospores and hyphae. The mycelium (hyphae) is the infective structure, which penetrates the plant's gynophore and initiates the infection. For this action, the expression of the Pepl protein would be necessary since it was identified in other Ustilaginomycetes such as Ustilago maydis and Ustilago hordei, which infect corn and barley, respectively, and its expression is essential for this action.
\end{abstract}


We were able to amplify the Pep1 DNAc of Thecaphora frezii whose translated sequence would code for a 180 amino acid protein. Great homologies with orthologous of other species and the presence of four conserved cysteines were observed. In parallel, we measured the expression levels of this transcript, being higher in the hyphae, coinciding with the infective stage of the fungus.

Future functional studies of gene inactivation of the pepl gene should be carried out to phenotypically verify the effect that this gene causes in peanut crops.

KeyWords: Thecaphora frezii; Pep1; peanut; infection.

\section{Introducción}

El maní (Arachis hypogaea L.) es uno de los cultivos leguminosos más importante del mundo, nativo de Sudamérica, distribuido en Brasil, Paraguay, Bolivia, Argentina y Uruguay ${ }^{1}$. De los 49.171 millones de toneladas estimadas mundialmente para la cosecha 2021, se observa que China, India, Nigeria, EEUU y Argentina se constituyen en los cinco principales productores, con el $37 \%, 12 \%, 9 \%, 6 \%$ y $3 \%$ de la producción mundial respectivamente ${ }^{2}$. Durante el ciclo de cultivo, el maní es atacado frecuentemente por enfermedades de origen fúngico especialmente algunas que se desarrollan en el suelo durante la etapa de formación del fruto, durante la cosecha y en las fases de secado y almacenamiento del grano $^{3,4}$. Uno de los causantes es el hongo Thecaphora frezii (T. frezii) $)^{5}$.

El carbón del maní, enfermedad causada por $T$. frezii, un hongo biótrofo, produce numerosas pérdidas anuales, teniendo mayor incidencia en la zona sur de la provincia de Córdoba. El mismo, fue detectado por primera vez en la campaña 1994/95, mientras que su prevalencia, incidencia y severidad se ha ido incrementando en los últimos 10 años, expandiéndose, además, a otras provincias $^{6}$.

Ustilago maydis ( $U$. maydis) se considera un modelo fúngico importante que ha sido ampliamente utilizado en estudios biológicos y genéticos ${ }^{7}$ y se ha considerado como un hongo de gran importancia para el estudio del desarrollo dimórfico y de interacción planta-patógeno. La relación entre $T$. frezii y $U$. maydis, es que este último hongo también pertenece a la clase de Ustilagomicetes y genera carbón en las plantas de maíz.

Se acepta que la mayoría de los genes de avirulencia fúngica codifican factores de virulencia que se llaman efectores. La mayoría de los efectores fúngicos se secretan, siendo proteínas ricas en cisteína, y ha sido demostrado un papel en la virulencia de algunos de ellos $^{8}$. U. maydis expresa un efector llamado Pepl (Protein essential during penetration 1$)^{9}$. El gen pepl está específicamente expresado durante el desarrollo del patógeno U. maydis ${ }^{9}$. En este hongo, variantes mutantes delecionadas del gen pepl formaban estructuras de penetración normales pero la infección se detenía inmediatamente después de la penetración epidérmica. Además, se encontró que Pepl se localiza en el apoplasto de la planta donde se acumula particularmente en sitios de pasajes de célula a célula de hifas biotróficas de U. maydis ${ }^{9}$. Pepl no solo es esencial para la virulencia de $U$. maydis sino también para el hongo relacionado con el carbón de cebada, Ustilago hordei (U. hordei), que indica una función conservada de Pepl en otros biótrofos fúngicos además de U. maydis ${ }^{9}$.

\section{Objetivo}

El objetivo de este trabajo fue comenzar a identificar y caracterizar a la proteína Pepl, cuya actividad sería fundamental para la infección por parte de $T$. frezii sobre las plantas de maní.

\section{Material y métodos}

\section{Aislamiento y cultivo de Thecaphora frezii}

Las teliosporas $T$. frezii fueron obtenidas de cajas de maní que presentaban síntomas de enfermedad (hipertrofia) y descontaminadas superficialmente con etanol. Las teliosporas se desinfectaron con hipoclorito de sodio $5 \%$ durante 5 min y se lavaron 3 veces con agua destilada estéril durante 10 minutos. Las hifas fueron obtenidas por germinación de las teliosporas en medio PDA (Potato Dextrose Agar). Las basidiosporas se obtuvieron a partir de las hifas crecidas en medio sólido, las cuales se sembraron en agar agua (1,5 $\%$ agar-agar). Se corroboró la formación de dichas estructuras mediante microscopía óptica.

\section{Aislamiento de ARN y síntesis de ADNc}

El ARN total de los tres estadios de T. frezii (se combinaron tres cultivos independientes para cada etapa) se extrajo usando TRIzol (Invitrogen, EE. UU.) siguiendo las recomendaciones del fabricante. $\mathrm{El} \mathrm{ADNg}$ se eliminó mediante digestión en columna con ADNasa (Qiagen) al 
doble de la concentración recomendada por el fabricante. Se controló la posible degradación y contaminación del ARN en un gel de agarosa al $1,5 \%(\mathrm{p} / \mathrm{v})$ y se confirmó la pureza del ARN usando el espectrofotómetro NanoPhotometer (IMPLEN, CA, EE. UU.). La concentración de ARN se midió con el kit de ensayo de ARN Qubit y el fluorómetro Qubit 2.0 (Life Technologies, CA, EE. UU.).

La síntesis de la primera hebra de ADNc se realizó con $1 \mu \mathrm{L}$ de Oligo-(dT)20 (50 $\mu \mathrm{M})$ (Invitrogen), 1 $\mu \mathrm{g}$ de ARN total y $400 \mathrm{U}$ de SuperScript III RT (transcriptasa inversa, Invitrogen) en un volumen de reacción de $20 \mu \mathrm{L}$ incubado a $55^{\circ} \mathrm{C}$ durante $1 \mathrm{~h}$.

\section{Amplificación y secuenciamiento del ADNc de pep1}

La amplificación rápida de los extremos del ADNc 5 'y 3' (5'-RACE y 3'-RACE) se realizó utilizando el kit comercial GeneRacer (Invitrogen) según las indicaciones del fabricante. Las secuencias de los oligonucleótidos fueron: 5'-RACE-GSP1 5' AYRCAVGTYTGSGGNAG 3'; 5'-RACE-GSP2 5' TTGTTKKCKVDRTCGTA 3'; 5'-RACEGSP3 5' CYTGRDVBKYGCAGT 3'; 3'-RACEGSP1 5' TACGAYHBMGMMAACAA 3'; 3'RACE-GSP2 5' CTNCCSCARACBTGYRT 3'. Después de la electroforesis de los productos de PCR, se cortaron dos bandas correspondientes al tamaño esperado de aproximadamente $280 \mathrm{pb}$ (5' RACE) y 260 pb (3'-RACE) del gel de agarosa y se purificaron usando el kit de purificación de PCR QIAquick (QIAGEN, Hilden, Alemania). A continuación, los productos de PCR se secuenciaron en un secuenciador de ADN automatizado ABI 3130XL (Applied Biosystems, Foster City, CA, EE. UU.).

\section{Análisis de la secuencia del ADNc de pep1}

La secuencia del ADNc de pep1 de T. frezii se comparó con las de otros hongos depositados en GenBank utilizando las herramientas "BLAST-N" o "BLAST-X" disponibles en el sitio web del Centro Nacional de Información Biotecnológica (NCBI). La secuencia de aminoácidos de Pep1 de T. frezii se dedujo del ADNc correspondiente utilizando la herramienta de traducción del sitio web ExPASy Proteomics (https://web.expasy.org/translate/). La presencia de péptido señal fue detectada usando el predictor SignalP-5.0 Server (http://www.cbs.dtu.dk/services/SignalP/). La predicción de efector fúngico en secreciones se realizó a través del programa EffectorP, que ha sido diseñado para distinguir proteínas secretadas de efectores secretados en hongos patógenos de plantas (http://effectorp.csiro.au/).

\section{Comparación de secuencias y relación filogenética}

Las secuencias de aminoácidos completas se alinearon utilizando el programa Clustal W (https://www.ebi.ac.uk/Tools/msa/clustalo/). Las secuencias de Pepl utilizadas para la comparación fueron de las siguientes especies (número de acceso de GenBank entre paréntesis): Anthracocystis flocculosa (A. flocculosa) PF-1 (XP_007878051.1), U. maydis 521 (XP_011387901.1), U. hordei (XP_041411115.1) y Kalmanozyma brasiliensis (K. brasiliensis) (XP_016292919.1). Para deducir la secuencia de Pepl de Thecaphora thlaspeos (T. thlaspeos) fue necesario utilizar los programas tBLASTn (https://blast.ncbi.nlm.nih.gov/Blast.cgi?PROGR $\mathrm{AM}=$ tblastn\&PAGE_TYPE $=$ BlastSearch\&LINK _LOC=blasthome) y http://bioinf.unigreifswald.de/augustus/submission.php

(UWYS01000010.1). El análisis filogenético fue realizado utilizando las condiciones por defecto del software disponible en http://www.phylogeny.fr/simple_phylogeny.cgi.

\section{Cuantificación de los transcriptos de pep1 de acuerdo al estadio de $T$. frezii}

Se realizó la cuantificación de la expresión génica de pepl en los tres estadios de T. frezii a través del sistema de detección de PCR en tiempo real StepOne Plus Real-Time PCR system ${ }^{\circledR}$ (ThermoFisher, Massachusetts, EE. UU.). Los oligonucleótidos se diseñaron utilizando el programa accesible en la página de GenScript ${ }^{\circledR}$ (https://www.genscript.com/tools/real-time-pcrtaqman-primer-design-tool) (Tabla 1).

El ADNc se preparó a partir de las mismas muestras de ARNs utilizadas para el análisis de 5'RACE y 3'-RACE.

La expresión génica relativa se realizó usando el transcripto de actina como gen de referencia para la normalización de la expresión. La especificidad de los amplicones se verificó mediante el análisis de las curvas de fusión y mediante el secuenciamiento de los fragmentos obtenidos. El cambio de expresión en el gen pepl, en relación con la expresión de actina se calculó utilizando el método $2^{-\triangle \Delta C T}{ }^{10}$. La media y $\operatorname{DS}( \pm)$, fueron entonces calculadas para cada una de las diferentes muestras. Los datos fueron analizados con el test de Student para establecer las diferencias significativas. 
Tabla 1. Secuencias de oligonucleótidos usadas en la Real Time PCR

\begin{tabular}{lcc}
\hline Nombre & Secuencia $\left(5^{\prime} \rightarrow 3^{\prime}\right)$ & Tamaño esperado del amplicón $(\mathrm{pb})$ \\
\hline pepl-F & CATCCTCCTCCTCACGTACC & 141 \\
pepl-R & GCCTTCTGCTGGTTGTTGAA & \\
\hline actina-F & CTACGTTGCCCTCGACTTTG & 107 \\
actina- $\mathrm{R}$ & CGTTTCCGACAGTGATGACC & \\
\hline
\end{tabular}

\section{Resultados}

\section{Características de la secuencia de pep1}

A partir del ADNc de $T$. frezii se logró amplificar un fragmento de $543 \mathrm{pb}$ correspondientes a la totalidad del transcripto del gen pepl. La secuencia fue registrada en GenBank bajo el código MZ337396 y codificaría para una proteína de 180 aminoácidos, compatible con los tamaños observados en otras proteínas ortólogas.

Paralelamente se analizó en la secuencia proteica predicha la posible presencia de un péptido señal a través del predictor SignalP-5.0, el cual arrojó una probabilidad del 0,9062 de presencia del mismo. Además, para evaluar la posibilidad de que la proteína deducida pudiera actuar como efector, la analizamos mediante el programa EffectorP, asignando una probabilidad del 0,667; 0,802; 0,555 y 0,682 de presentar esa función para $T$. frezii; U. maydis; U. hordei y para T. thlaspeos, respectivamente.

\section{Alineamientos de secuencias Pep1 y relación filogenética}

Para estos análisis, se alinearon las secuencias de proteínas ortólogas de Pepl de hongos filogenéticamente próximos a $T$. frezii. Para ello se compararon las secuencias de Pepl de U. maydis 521, U. hordei, K. brasiliensis, T. thlaspeos y A. flocculosa PF1 (Figura 1A y Tabla 2). Se consideró que las proteínas eran homólogas si compartían un $25 \%$ o más de identidad con una longitud de alineación mayor a 80 aminoácidos ${ }^{11}$. En la Figura 1B observamos la estructura de la proteína Pepl de $T$. frezii, con su péptido señal y sus 4 cisteínas conservadas.

A

T. frezij

T. thlaspeos

A. flocculosa PF1

U. maydis 521

U. hordei

K. brasiliensis

T. $f r e z i j$

T. thlaspeos

A. flocculosa PF1

U. maydis 521

$U$. hordei

K. brasiliensis

T. frezij

T. thlaspeos

A. flocculosa PF1

U. maydis 521

U. hordei

K. brasiliensis

T. $f_{r e z i i}$

T. thlaspeos

A. flocculosa PF1

U. maydis 521

U. hordei

K. brasiliensis

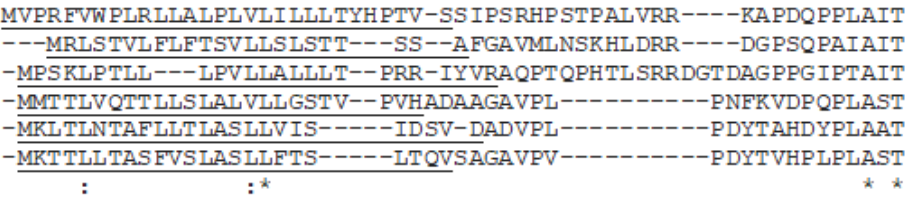

MFWFNNOQKA TCPDLTARVKSVTGYTICDFODDYDSANNSYFLPO CVILIA PLSEALFNI L FWFNNI ELA I CY DMR DRVES I SEI I C NAQDDY DRINNTYALPQ TCI PLI PRSERLHSQ L FWFNNLEVG I C DMMRRVVSD PDS FDCNAQGDYDRDNNTYALPQ TCEALI PKSEALLYR FYWFSSVEVGVCYNPQARVGSI KGALH CTHQENY DRDNNSYTLPQ7CVALK PLGKAFSSN FYWFSSVEVGV Y SPRARVGSI KGALHCTHOFNYDIENNTWTIPOTCVALK PLGEPLSNA FYWF SSVEVGVCYSPTARVASI PGALH C THQEKYDIDNNSWTLPQ IC VALK PLGGPLSSA 104

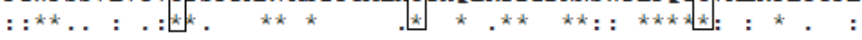

ISFA RRVG-TLGEMDVKGNTPSTPITPGNPGLGGMPGTNPYAOSGNP----GLGGLPG-VKMACKEAAGTFG ILV---RPSA---------NDGVVDSNQS POPO------------I TQA CRQTNGKFDTLT---APPG---------TDMNP GPAAASGAGAA---GTSASAA-VRDS CFNAKG I FNVIV PAS SNAL------------GSQAY DAVQAKGGTGGTG---VRDSCFNARG SFNLI I PAS SNAD--------------GSQAYNAI SNKGADGAASDPGSQ VRDS KKNAKG TFNVIT PAG SNAE-----------GGQAYDAIQKGGPGGGGAGAGGD $: \quad:$ : : : : :

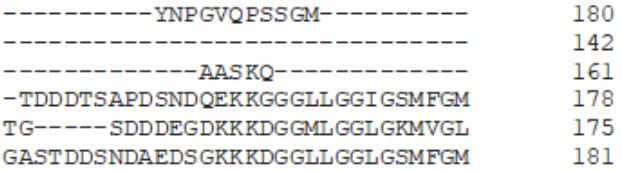

B

MVPRFVWPLRLLALPLVLILLLTYHPTVSSIPSRHPSTPALVRRKAPDQPPLAITMFWFNNQQKAI CYDLTARVKSVTGYTDCDFQDDYDSANNSYFLPQTCVLIAPLSEALFNILSEACRKVGTLGEMD VKGNTPSTPITPGNPGLGGMPGTNPYAQSGNPGLGGLPGYNPGVQPSSGM

Figura 1. A: Alineamiento de secuencias de Pep1 de T. frezii, T. thlaspeos, A. flocculosa PF1, U. maydis 521, U. hordei y $K$. brasiliensis. En rectángulos se señalan las cuatro cisteínas conservadas. *, identidad de secuencia en todo el conjunto de la alineación; :, una sola discrepancia en el conjunto de la alineación;., dos discrepancias en la alineación. Las secuencias subrayadas corresponden a los péptidos señales. B: Secuencia completa de la proteína Pepl de T. frezii señalando al péptido señal (subrayado) y a las 4 cisteínas conservadas (en negritas y sombreadas).

Revista Methodo: Investigación Aplicada a las Ciencias Biológicas. Universidad Católica de Córdoba. Jacinto Ríos 571 Bo Gral. Paz. X5004FXS. Córdoba. Argentina. Tel.: (54) 3514517299 / Correo: methodo@ucc.edu.ar / Web: methodo.ucc.edu.ar | ARTICULO ORIGINAL Rev. Methodo 2021;6(4):155-161. 
Tabla 2. Porcentaje de identidad y homología a nivel proteico de las distintas Pepl de hongos fitopatogénicos (excepto A. flocculosa) respecto Thecaphora frezii

\begin{tabular}{|c|c|c|c|}
\hline Hongo & $\mathrm{n}^{\circ}$ de aminoácidos deducidos & Identidad (\%) & $\begin{array}{c}\text { Homología } \\
(\%)\end{array}$ \\
\hline Thecaphora frezii & 180 & 100 & 100 \\
\hline $\begin{array}{c}\text { Anthracocystis flocculosa } \\
\text { PF1 }\end{array}$ & 161 & 47 & 63 \\
\hline Thecaphora thlaspeos & 142 & 44 & 62 \\
\hline Ustilago maydis 521 & 178 & 44 & 65 \\
\hline Ustilago hordei & 175 & 34 & 48 \\
\hline Kalmanozyma brasiliensis & 181 & 42 & 61 \\
\hline
\end{tabular}

Una posible relación evolutiva entre los hongos alineados anteriormente pudo observarse a través de la construcción de un árbol filogenético basados en las secuencias proteicas de Pepl (Figura 2).

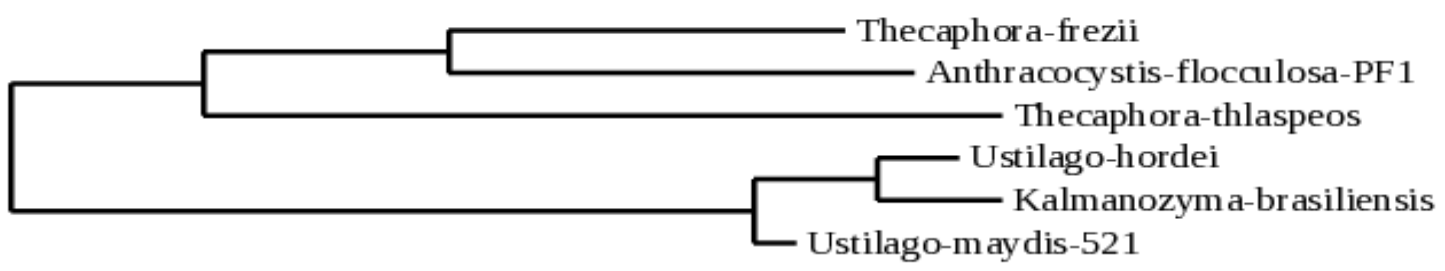

0.3

Figura 2. Árbol filogenético de Pepl construido en base a T. frezii, U. maydis 521, U. hordei, K. brasiliensis, T. thlaspeos y A. flocculosa PF1.

\section{Análisis de la expresión de pep1}

Los niveles de expresión del ARNm de pepl fueron cuantificados mediante PCR en Tiempo Real en los tres estadios del T. frezii, es decir, teliosporas, basidiosporas e hifas. Coincidentemente con lo esperado, los niveles del transcripto de pepl fueron más elevados en las hifas y basidiosporas, respecto a lo que sucede en teliosporas (Figura 3). Se calcularon las medias, desviaciones standart y el valor de $\mathrm{p}$ mediante el test de Student. La diferencia de expresión no fue significativa cuando se compararon los niveles presentes en las basidiosporas vs. hifas ( $p>0,05)$, mientras que la diferencia fue significativa entre basidiosporas vs. teliosporas e hifas vs. teliosporas ( $\mathrm{p}<0,001)$.

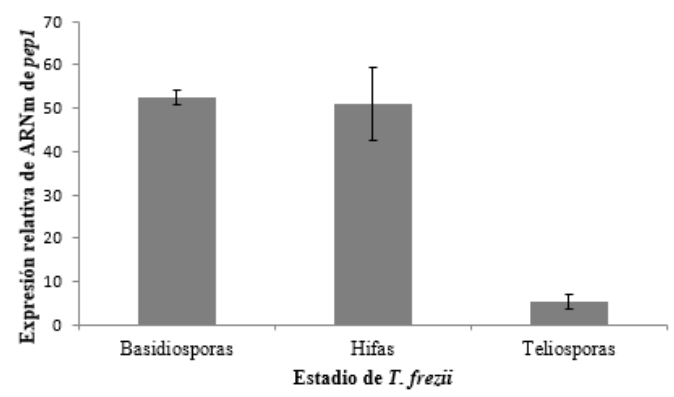

Figura 3. Niveles de expresión del ARNm de pepl en los tres estadios de T. frezii. La media y DS ( \pm ), fueron calculadas para cada una de las diferentes muestras.

\section{Discusión y Conclusiones}

Uno de los microorganismos que infecta a los cultivos del maní es el hongo $T$. frezii. Esta afección, llamada carbón del maní, produce enormes pérdidas en estos cultivares.

Para que muchos hongos sean fitopatogénicos es necesario la expresión de efectores que desencadenan el proceso infeccioso ${ }^{12}$, siendo uno de ellos la Pepl (Protein essential during penetration 1$)^{9}$.

Uno de los hongos que presenta varias características comunes con $T$. frezii es el hongo U. maydis, que además de ser usado como modelo de estudio de muchas características de hongos fitopatogénicos, causa la enfermedad del carbón

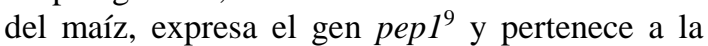
misma clase de hongos que T. frezii ${ }^{13}$.

En este trabajo nos propusimos caracterizar al menos de manera parcial a la proteína Pepl de $T$. frezii.

A partir de la obtención del ADNc de pepl, procedimos a la secuenciación del mismo y a la deducción de la secuencia aminoacídica correspondiente. Los análisis de esta secuencia mostraron la posible presencia de un péptido señal, el cual se encuentra presente en todos los efectores $^{8}$. Además, realizando un análisis de homología con ortólogos de Pepl de hongos próximos filogenéticamente con $T$. frezii, pudimos 
encontrar ciertas regiones conservadas, particularmente la presencia de cuatro cisteínas que ya se ha demostrado que son esenciales para la función $P e p 1^{14}$. Esta estructura proteica también se encuentra presente en hongos que afectan a plantas monocotiledóneas como dicotiledóneas, entre otros, Sporisorium reilianum; Sporisorium scitamineum; Ustilago avenae; Ustilago nuda y Melanopsichium pennsylvanicum ${ }^{14}$.

Otro aspecto importante que encontramos es que la presencia del transcripto de pepl está altamente expresado en estructuras del hongo como son las basidiosporas e hifas, estando estas últimas estructuras asociadas al estadio infectivo del hongo ${ }^{15}$.

Los aportes descriptos en el presente trabajo, pueden ser el puntapié inicial para comenzar a identificar factores que están involucrados en el proceso infeccioso de $T$. frezii que lleva a la enfermedad del carbón del maní y a las consecuentes pérdidas económicas.

\section{Bibliografía}

1. Krapovickas A, Gregory WC. Taxonomía del género Arachis (Leguminosae). Bonplandia [Internet]. 2007 Jan 1; 16:1. Available from: http://revistas.unne.edu.ar/index.php/bon/arti cle/view/158

2. United States Department of Agriculture. Peanut Explorer [Internet]. web site. 2021 [cited 2021 Jul 8]. Available from: https://ipad.fas.usda.gov/cropexplorer/cropvi ew/commodityView.aspx? cropid $=2221000$

3. Pitt JI, Dyer SK, McCammon S. Systemic invasion of developing peanut plants by Aspergillus flavus. Lett Appl Microbiol [Internet]. 1991 Jul;13(1):16-20. Available from: http://doi.wiley.com/10.1111/j.1472765X.1991.tb00558.X

4. Fernandez EM, Rosolem CA, Maringoni AC, Oliveira DMT. Fungus incidence on peanut grains as affected by drying method and $\mathrm{Ca}$ nutrition. F Crop Res [Internet]. 1997 May;52(1-2):9-15. Available from: https://linkinghub.elsevier.com/retrieve/pii/S 0378429096034612

5. Marinelli A, March GJ, Oddino C. Aspectos biológicos y epidemiológicos del carbón del maní (Arachis hypogaea L.) causado por Thecaphora frezii Carranza \& Lindquist. AgriScientia [Internet]. 2008;25(1):1-5. Available from: http://www.scopus.com/inward/record.url?ei $\mathrm{d}=2$-s 2.0 77950144062\&partnerID=tZOtx3y1
6. Cazzola, N; Gateu, M.; March, G.; Marinelli, A.; García, J. J.; Rago, A. y Oddino C. Intensidad y pérdidas ocasionadas por carbón del maní según regiones de producción. XXVII Jorn Nac Maní. 2012;34-5.

7. Bölker M. Ustilago maydis - a valuable model system for the study of fungal dimorphism and virulence. Microbiology [Internet]. 2001 Jun 1;147(6):1395-401. Available from: http://mic.microbiologyresearch.org/content/j ournal/micro/10.1099/00221287-147-6-1395

8. Stergiopoulos I, de Wit PJGM. Fungal Effector Proteins. Annu Rev Phytopathol [Internet]. 2009 Sep;47(1):233-63. Available from:

http://www.annualreviews.org/doi/10.1146/a nnurev.phyto.112408.132637

9. Doehlemann $\mathrm{G}$, van der Linde K, Aßmann D, Schwammbach D, Hof A, Mohanty A, et al. Pep1, a Secreted Effector Protein of Ustilago maydis, Is Required for Successful Invasion of Plant Cells. Howlett BJ, editor. PLoS Pathog [Internet]. 2009 Feb 6;5(2): e1000290. Available from: https://dx.plos.org/10.1371/journal.ppat.1000 290

10. Livak KJ, Schmittgen TD. Analysis of Relative Gene Expression Data Using RealTime Quantitative PCR and the $2-\Delta \Delta C T$ Method. Methods [Internet]. 2001 Dec;25(4):402-8. Available from: https://linkinghub.elsevier.com/retrieve/pii/S 1046202301912629

11. Sander C, Schneider R. Database of homology-derived protein structures and the structural meaning of sequence alignment. Proteins Struct Funct Genet [Internet]. 1991 Jan;9(1):56-68. Available from: http://doi.wiley.com/10.1002/prot.340090107

12. Selin C, de Kievit TR, Belmonte MF, Fernando WGD. Elucidating the Role of Effectors in Plant-Fungal Interactions: Progress and Challenges. Front Microbiol [Internet]. 2016 Apr 27; 7:600. Available from: www.frontiersin.org

13. Boekhout T, Fonseca Á, Sampaio JP, Bandoni RJ, Fell JW, Kwon-Chung KJ. Discussion of Teleomorphic and Anamorphic Basidiomycetous Yeasts. In: The Yeasts [Internet]. Elsevier; 2011. p. 1339-72. Available from: https://linkinghub.elsevier.com/retrieve/pii/B 9780444521491001002 
14. Hemetsberger C, Mueller AN, Matei A, Herrberger C, Hensel G, Kumlehn J, et al. The fungal core effector Pep1 is conserved across smuts of dicots and monocots. New Phytol [Internet]. 2015 May 27;206(3):1116-26. Available from: https://onlinelibrary.wiley.com/doi/10.1111/n ph.13304

15. Sinha M, Shree A, Singh K, Kumar K, Singh SK, Kumar V, et al. Modulation of fungal virulence through CRZ1 regulated F-BARdependent actin remodeling and endocytosis in chickpea infecting phytopathogen Ascochyta rabiei. Stukenbrock EH, editor. PLOS Genet [Internet]. 2021 May 17;17(5): e1009137. Available from: https://doi.org/10.1371/journal.pgen.1009137

\section{(c) (1)(0) BY NC SA}

